\title{
ARRHENIUS JUMP RATE OF MUONS IN SOME NON-STOICHIOMETRIC METALLIC AND INTERMETALLIC HYDRIDES
}

\author{
G.C. Млнато \\ R.K.M.V.C. College, Rahara, 24 Parganas (North), West Bengal, India \\ (Received March 15, 1994)
}

\begin{abstract}
Using the energy loss rate in the process $\mu^{+}+\mathrm{e}^{-} \rightarrow \nu_{\mathrm{e}}+\bar{\nu}_{\mu}$ we develop a model which formulates the jump rate for muon diffusion in non-stoichiometric metallic hydrides $\mathrm{LaH}_{2.75}, \mathrm{CeH}_{x}, \mathrm{YH}_{2}$ and intermetallic liydrides $\mathrm{ZrV}_{2} \mathrm{H}_{x}$, $\mathrm{Zr}_{2} \mathrm{NiH}_{x}, \mathrm{HI}_{2} \mathrm{CoH}_{3}, \mathrm{Cu}_{0.5} \mathrm{Ni}_{0.5} \mathrm{H}_{0.72}$ and $\mathrm{Ni}_{0.35} \mathrm{Ti}_{0.65} \mathrm{H}_{1.5}$. The jump rate is found to follow an Arrhenius-like relation over some temperature ranges.

PACS numbers: $66.30 . \mathrm{Jt}$
\end{abstract}

\section{Introduction}

In recent years the diffusion of muon in non-stoichiometric metallic and intermetallic hydrides has become a very interesting and important problem for both the experimental observation and theoretical analysis. In the non-stoichiometric hydrides considered here the hydrogen concentration is very high. Then the majority of the preferential interstitials are occupied by the hydrogen; the implanted muon cannot find any connected path through the lattices at low temperatures; the muons manage to find a few remaining sites, but their subsequent motion is blocked unless the hydrogens themselves are moving. Therefore at low temperatures the muons are observed to remain in a stationary state. At higher temperatures the muons are better to find sites vacated by hydrogen atoms; and this shows the hydrogen diffusion and muon diffusion very similar with equal or nearly equal diffusion parameters.

In our earlier papers [1-4] the jump rate for muon diffusion in metals and non-stoichiometric metallic and intermetallic hydride-targets has been formulated in a newly developed model. This article is an extension to our previous works and presents some more results for the jump rate of muons in non-stoichiometric metallic hydrides like $\mathrm{LaII}_{2.75}, \mathrm{CeI}_{x}, \mathrm{YII}_{2}$ and intermetallic hydrides like $\mathrm{ZrV}_{2} \mathrm{H}_{x}$, $\mathrm{Zr}_{2} \mathrm{NiII}_{x}, \mathrm{IIf}_{2} \mathrm{CoH}_{3}, \mathrm{Cu}_{0.5} \mathrm{Ti}_{0.5} \mathrm{II}_{0.72}$ and $\mathrm{Ni}_{0.65} \mathrm{Ti}_{0.35} \mathrm{II}_{1.5}$. The jump rates for muon diffusion in these targets show an Arrhenius-like relation over some appropriate temperature ranges. 


\section{Theory}

The following assumptions are made for the development of the present model:

A wide beam of polarized and highly energetic muons is made incident upon the target so that a large muonic flux penetrates into the target. The muons, upon entering the target, are considered to form a "muon gas" in which all the muons can move independently obeying the Fermi distribution law.

The muon gas diffuses through an "electron gas" offered by the target. In case of metals and metallic compounds this electron gas is formed by the valence electrons of the target, whilc in case of non-stoichiometric metallic and intermetallic hydrides the electron gas is formed by the non-bonding or unpaired electrons of the target.

Due to the very high velocity of the muons $[5]\left(\boldsymbol{P}_{\mu} \approx 105 \mathrm{MeV} / c\right)$ and the high density of electrons $[6,7]$ prevailing in the electron gas so formed, the muon cannot form a muonium atom and so while diffusing through the electron gas the muons behave as if they were free particles.

During the diffusion througl the electron gas a muon visits many sites by performing jumps and finally disappears in a muon-electron annililation process represented by

$$
\mu^{+}+\mathrm{e}^{-} \rightarrow \nu_{\mathrm{e}}+\bar{\nu}_{\mu} .
$$

It is further assumed that the energy necessary to produce such an annililation process corresponds to the activation energy for the diffusion of muon through the electron gas.

While jumping over the intervening barricrs through the electron gas the rnuon finds an electron to collide and results in the annihilation process in which energy is liberated due to neutrino emission. In such a picture, the jump rate for muon diffusion becomes proportional to this energy loss rate which is liberated from the target. Therefore, the jump rate for muon diffusion is written in the form

$$
\frac{1}{\tau}=F(z) \frac{\mathrm{d} U}{\mathrm{~d} t}\left[\mathrm{~s}^{-1}\right]
$$

where $\mathrm{d} U / \mathrm{d} t$ represents the energy loss rate which is liberated from the target, $F(z)$ denotes a constant of proportionality depending upon the target only.

Thus, using the encrgy loss rate from the target an attempt lias been made to formulate the jump rate for muon diffusion in a variety of targets like metals of different crystallographic structures and magnetic origins, metallic compounds and non-stoichiometric metallic and intermetallic hydrides of varying hydrogen concentrations. The model aims at framing a theory which formulates the jump rate for muon diffusion in a systematic, generalized and simplified way for all the targets most commonly used in the present days.

\subsection{Energy loss rale $(\mathrm{d} U / \mathrm{d} l)$}

The energy loss rate liberated from the target is given by

$$
-\frac{\mathrm{d} U}{\mathrm{~d} t}=\left(E_{\mu+}+E_{-}\right) \sigma n_{+} n_{-} v_{\text {rel }}\left[\operatorname{erg} \mathrm{cm}^{-3} \mathrm{~s}^{-1}\right] \text {, }
$$


where $E_{\mu}+$ represents the total encrgy of the muon, $E_{-}$represents the total energy of the electron, $n_{+}$is the number density of muons, $n_{-}$is the number density of electrons, $v_{\text {rel }}$ is the relative velocity between the incident muon and the electron of the elcctron gas of the target and $\sigma$ is the cross-section of the reaction (1).

\subsubsection{Cross-section of the reaclion $(\sigma)$}

The Feynman diagram of the reaction is shown in Fig. 1.

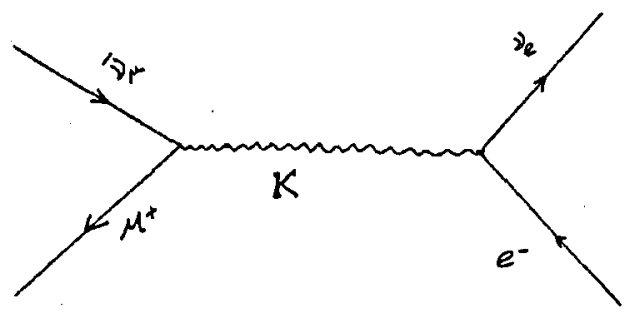

Fig. 1. Feynman diagram of the reaction (1). $K$ is the momentum of the virtual photon.

This is a leptonic weak process involving four leptons and is studied by a standard electroweak thcory. The expression for cross-section is

$$
\sigma=\frac{4 g^{4}}{\pi c^{4}} \frac{\left|\boldsymbol{P}_{\nu}\right|^{3}\left(E_{\mu^{+}}-\left|\boldsymbol{P}_{\mu}\right| c\right)}{\left(E_{\mu+}+E_{-}-\left|\boldsymbol{P}_{\mu+}\right| c\right) K^{4} E_{\mu^{+}}}\left[\mathrm{cm}^{2}\right] .
$$

IIere $g$ is the photon-neutrino weak coupling constant and its value is $1.7 \times 10^{-6}$ [8], $K$ is the momentum of virtual photon and $c$ is the velocity of light.

For $\boldsymbol{P}_{\mu}=105 \mathrm{MeV} / c$ and $\boldsymbol{P}_{\nu}=74.9 \mathrm{MeV} / c$,

$$
\sigma=1.7 \times 10^{-40}\left[\mathrm{~cm}^{2}\right] \text {. }
$$

\subsubsection{The muon menter densily $\left(n_{+}\right)$}

In the muonic flux the number density of muons which diffuse through the electron gas resulting in the annihilation process is given by

$$
\begin{aligned}
n_{+} & =\frac{1}{\pi^{2} h^{3}} \int_{0}^{\alpha} \frac{\boldsymbol{P}_{\mu}^{2} \mathrm{~d} \boldsymbol{P}_{\mu}}{\exp \left(\frac{E_{\mu}+E}{k_{\mathrm{B}} T}+1\right)}=\frac{1}{\sqrt{2}}\left(\frac{m_{\mu} k_{\mathrm{B}}}{\pi h^{2}}\right)^{3 / 2} T^{3 / 2} \mathrm{e}^{-E / T} \\
& =1.4 \times 10^{19} T^{3 / 2} \mathrm{e}^{-E / T}\left[\mathrm{~cm}^{-3}\right] .
\end{aligned}
$$

IIere $E$ is the activation energy for the diffusion process which is also equal to the energy required to produce the annihilation process.

\subsubsection{The electron number densily ( $\left.n_{-}\right)$}

In these non-stoichiometric metallic or intermetallic non-stoichiometric hydride-targets the non-bonding electrons form the electron gas and the number density of such electrons is given by

$$
n_{-}=\frac{N d}{M}(v-x)\left[\mathrm{cm}^{-3}\right] \text {, }
$$


where $N$ represents the Avogadro number, $M$ and $d$ are respectively the molecular weight and density of the hydride-target, $v$ represents the number of valence electrons of the metal or the metallic cluster present in one molecule of the target and $x$ represents the hydrogen content in one molecule of the hydride-target.

\subsection{Jump rale for muon diffusion $(1 / \tau)$}

In some targets over-barrier diffusion of muon becomes predominant at high temperatures while at low temperatures diffusion is governed by a tunnelling process. For an over-barrier diffusion of muon, the constant $F(z)$ is suggested to have an empirical form

$$
F(z)=\frac{N M}{z^{2} d c^{2}}\left[\mathrm{erg}^{-1} \mathrm{~cm}^{3}\right] .
$$

IIere $z$ represents the sum of the atomic number of atoms present in one molecule of the stoichiometric hydride.

For an under-barrier tunnelling the proportionality constant $F(z)$ is to be multiplied by the potential barrier transparency coeflicient given by [9]:

$$
\beta=\exp \left[-\frac{2}{h}\left(2 m_{\mu} H\right)^{1 / 2} b\right] .
$$

Here $H$ and $b$ represent respectively the height and breadth of the potential barrier.

Therefore, finally the jump rate of muon in an over-barrier diffusion process is given by

$$
\frac{1}{\tau}=\frac{6.8 \times 10^{12}}{z^{2}}(v-x) \tau^{3 / 2} \mathrm{e}^{-E / T}\left[\mathrm{~s}^{-1}\right] .
$$

For an under-barrier diffusion process the above formula is multiplied by given in Eq. (9).

\section{Results}

The present model is now applied to formulate the jump rate for muon diflusion in the following hydride-targets. The activation energies are used from the respective experimental results. In these targets the implanted muons find that the majority of favourable sites are occupied by hydrogen atoms, the muons manage to find a few remaining empty sites a vailable for them but their subsequent motion is blocked unless the hydrogens themselves are moving [10]. Therefore at lower temperatures the muons are found practically immobile and at higher temperatures the muons are found to diffuse into sites vacated by the hydrogen atoms. Then, the motion of muon becomes identical with the hydrogen motion and is governed by identical or nearly identical diffusion parameters. Therefore, a knowledge of activation energy for hydrogen diffusion $\left(H_{\mathrm{p}}\right)$ throws much light on the activation energy for muon diffusion $(H)$. 


\section{1. $\mathrm{LaII}_{2.75}$}

Muon diffusion in this hydride has been studied over $15-300 \mathrm{~K}[11,12]$. It is found that the muons remain stationary up to $165 \mathrm{~K}$ above which the muons can diffuse in a tunnelling process. At high temperatures the muons residing on the same sublattice hop into the vacant sites created by the hydrogen jumps. Over 165-240 K the jump rate for muon diffusion follows an Arrhenius behaviour with an activation energy almost equal to that for hydrogen diffusion.

In the present model, considering the activation energy for muon diffusion $\approx 0.15 \mathrm{eV}[11,12]$, the muon is assumed to tunnel with an activation energy $1700 \mathrm{~K}$. Then, for the barrier width $b=1.2 \AA$, the potential barrier transparency coefficient $\beta$ becomes $4.4 \times 10^{-4}$ and the jump rate formula becomes

$$
\frac{1}{\tau}=2 \times 10^{5} T^{3 / 2} \mathrm{e}^{-1700 / T}\left[\mathrm{~s}^{-1}\right] .
$$

This formula very closely follows the experimental results over $165-240 \mathrm{~K}$.

$$
\text { 3.2. } \mathrm{Ce}_{x}(x=2.70,2.95)
$$

Muon diffusion in $\mathrm{CeH}_{x}$ is similar to that observed in $\mathrm{LaIH}_{2.75}$. In this hydride the muons remain static up to $\approx 180 \mathrm{~K}[13,14]$ and above this temperature the muons diffuse in a tunnelling process. For different values of $x$, the hydride target passes through different phases offering different hindrance to muon motion - in $\mathrm{CeH}_{2.70}$ and in $\mathrm{CeH}_{2.95}$ the activation energies for muon diffusion are $250 \mathrm{~K}$ and $800 \mathrm{~K}$ respectively.

In the present model, the muon is assumed to tunnel with an activation energy $800 \mathrm{~K}$. With the barrier width $b=2.6 \AA$, the barrier transparency coefficient $\beta$ becomes $2.3 \times 10^{-5}$ yielding the jump rate formula

$$
\frac{1}{\tau}=4.1 \times 10^{4}(3-x) T^{3 / 2} \mathrm{e}^{-800 / T}\left[\mathrm{~s}^{-1}\right] \text {. }
$$

This formula is found to be a good fit to the experimental results in both the phases of $\mathrm{CeH}_{x}$ over an approximate temperature range 180-240 K.

\section{3. $\mathrm{YII}_{2}$}

Muon diffusion in $\mathrm{YII}_{2}$ has been studied over the temperature range $16-600 \mathrm{~K}$ [15] and is found very similar to the hydrogen diffusion. Like the hydrogens, the muons are static at low temperatures and can diffuse at high temperatures. At $T>360 \mathrm{~K}$ the muons find sites vacated by hydrogen atoms and the muon motion becomes identical with hydrogen motion and is governed by the same diffusion parameters $[16,17]$.

Considering the hydrogen diffusion, in the present model muon is assumed to tunnel with a potential barrier height $400 \mathrm{meV}$. Then for the barrier width $\mathrm{b}=0.5 \AA$, the barrier transparency coefficient $\beta$ becomes $10^{-2}$. Therefore the jump rate formula becomes

$$
\frac{1}{\tau}=3.9 \times 10^{7} T^{3 / 2} \mathrm{e}^{-4640 / T}\left[\mathrm{~s}^{-1}\right] .
$$

This formula finds a good comparison to the experimental values at temperatures above $300 \mathrm{~K}$. 


$$
\text { 3.4. } Z r V_{2} H_{x}(x=3.25,3.6,4.0,4.8)
$$

Muon diffusion in $\mathrm{ZrV}_{2} \mathrm{II}_{x}$ has been studied over the temperature range $2-250 \mathrm{~K}$ [18]. In this study muon in found to remain immobile for $T<50 \mathrm{~K}$ above which the muons start to diffuse. For $50<T<150 \mathrm{~K}$ this diffusion remains confined to a limited number of sites, e.g., within clusters of proton vacancies. At higher temperatures, the muon diffuses through the sites vacated by hydrogen and the muon diffusion becomes identical with the hydrogen diffusion. Over $150-250 \mathrm{~K}$ the jump rate for muon diffusion is given by

$$
\begin{gathered}
\frac{1}{\tau}=(8 \pm 5) \times 10^{9} \exp \left[-\frac{(145 \pm 20) \mathrm{meV}}{k_{\mathrm{B}} T}\right]\left[\mathrm{s}^{-1}\right] \text { for } x=3.25,3.6,4.0 \\
=(8 \pm 5) \times 10^{9} \exp \left[-\frac{(100 \pm 20) \mathrm{meV}}{k_{\mathrm{B}} T}\right]\left[\mathrm{s}^{-1}\right] \text { for } x=4.8 .
\end{gathered}
$$

The dependence of activation energy on hydrogen concentration is related to the different electronic structures of the hydride.

The present model assumes the muon tunnel under a potential barrier $125 \mathrm{meV}$. Then for $b=1.3 \AA, \beta$ is $1.1 \times 10^{-3}$ yielding the jump rate

$$
\frac{1}{\tau}=8.8 \times 10^{5}(6-x) T^{3 / 2} \mathrm{e}^{-1450 / T}\left[\mathrm{~s}^{-1}\right] .
$$

This formula agrees well with the experimental results over the temperature range $150-250 \mathrm{~K}$.

$$
\text { 3.5. } 2 r_{2} \mathrm{NiH}_{x}(x=3.0,4.0,4.8)
$$

Muon diffusion in $\mathrm{Zr}_{2} \mathrm{NiII}_{x}$ up to about room temperature has been investigated by Baudry et al. [19] and Boyer at al. [20]. In $\mathrm{Zr}_{2} \mathrm{NiII}_{3}$ and $\mathrm{Zr}_{2} \mathrm{NiH}_{4.8}$ the muons are immobile at low temperatures; in $\mathrm{Zr}_{2} \mathrm{NiH}_{4}$ muons mianage to tunnel at temperatures below $200 \mathrm{~K}$. However, at higher temperatures the muons can diffuse in all these hydrides with the different diffusion parameters

$$
\begin{aligned}
\frac{1}{\tau}=(3.5 & \pm 1) \times 10^{11} \exp \left[-\frac{(240 \pm 10) \mathrm{meV}}{k_{\mathrm{B}} T}\right]\left[\mathrm{s}^{-1}\right] \text { for } x=3.0 \\
& =(1.7 \pm 0.6) \times 10^{10} \exp \left[-\frac{(220 \pm 10) \mathrm{meV}}{k_{\mathrm{B}} T}\right]\left[\mathrm{s}^{-1}\right] \text { for } x=4.0 \\
& =(3 \pm 1) \times 10^{11} \exp \left[-\frac{(310 \pm 20) \mathrm{meV}}{k_{\mathrm{B}} T}\right]\left[\mathrm{s}^{-1}\right] \text { for } x=4.8
\end{aligned}
$$

The jump rate for muon diffusion in $\mathrm{Zr}_{2} \mathrm{NiII}_{4}$ below $200 \mathrm{~K}$ is described by

$$
\frac{1}{\tau}=(2.3 \pm 0.9) \times 10^{6} \exp \left[-\frac{(38 \pm 6) \mathrm{meV}}{k_{\mathrm{B}} T}\right]\left[\mathrm{s}^{-1}\right] .
$$

In the present model, the potential barrier for muon diffusion $(H)$ is considered from a knowledge of the activation encrgy for hydrogen diffusion $\left(H_{\mathrm{p}}\right)$ in these hydrides. Using $H_{\mathrm{p}}=380 \pm 20 \mathrm{meV}$ [21] the value of $H$ is set at $330 \mathrm{meV}$. Therefore the jump rates of muon diffusion in all these hydrides are given by

$$
\frac{1}{\tau}=5.2 \times 10^{8}(6-x) T^{3 / 2} \mathrm{e}^{-3880 / T}\left[\mathrm{~s}^{-1}\right] .
$$


This formula agrees well with expcrimental jump rate values above $200 \mathrm{~K}$. Below this temperature muon in $\mathrm{Zr}_{2} \mathrm{NiII}_{4}$ is assumed to tunnel with an activation energy $30 \mathrm{meV}$. Then with $H=330 \mathrm{meV}$ and $b=1.6 \AA, \beta$ becomes $1.3 \times 10^{-6}$ and the jump rate formula is

$$
\frac{1}{\tau}=1.3 \times 10^{3} T^{3 / 2} \mathrm{e}^{-440 / T}\left[\mathrm{~s}^{-1}\right] .
$$

This formula is a good fit to the experimental results in $\mathrm{Zr}_{2} \mathrm{NiH}_{4}$ hydride below $200 \mathrm{~K}$.

\section{6. $\mathrm{Hf}_{2} \mathrm{CoH}_{3}$}

Muon diffusion in this hydride is qualitatively similar to that observed in $\mathrm{Zr}_{2} \mathrm{NiII}_{4}$ [22]. The muon becomes localized at interstitial sites at low temperatures up to $100 \mathrm{~K}$ above which the muon can diffuse in a tunnelling process. The motion of muon is strongly related to the motion of hydrogen atoms present in the target. Over the temperature rangc $100-250 \mathrm{~K}$ the jump rate for muon diffusion is given by

$$
\frac{1}{\tau}=3.2(3) \times 10^{6} \exp \left[-\frac{(32 \pm 8) \mathrm{meV}}{k_{\mathrm{B}} T}\right]\left[\mathrm{s}^{-1}\right] .
$$

Above $250 \mathrm{~K}$ muon diffusion and hydrogen diffusion become identical governed by the same activation energy $\approx 240 \mathrm{meV}$ [22]. Muon diffusion may be considered as an over-barrier hopping process with a rate

$$
\frac{1}{\tau}=4.6(1) \times 10^{10} \exp \left[-\frac{(260 \pm 20) \mathrm{meV}}{k_{\mathrm{B}} T}\right]\left[\mathrm{s}^{-1}\right] .
$$

In the present theory the potential barrier for muon diffusion $(H)$ is set at $240 \mathrm{meV}$. Then the over-barrier jump rate of muon is given by

$$
\frac{1}{\tau}=2.7 \times 10^{8} T^{3 / 2} \mathrm{e}^{-2780 / T}\left[\mathrm{~s}^{-1}\right] .
$$

This formula agrees well with the experimental results over 250 to about the room temperature.

At low temperature the muon is assumed to tunnel with an activation energy $32 \mathrm{meV}$. For $I I=240 \mathrm{meV}$ and $b=1.6 \AA, \beta$ becomes $10^{-5}$ and the jump rate in the tunnelling process becomes

$$
\frac{1}{\tau}=2.7 \times 10^{3} T^{3 / 2} \mathrm{e}^{-390 / T}\left[\mathrm{~s}^{-1}\right] .
$$

This formula closely follows Eq. (20) over the temperature interval 100-250 K.

\section{7. $C u_{0.5} T i_{0.5} H_{0.72}$}

In a study on muon diffusion in this amorphous intermetallic hydride the muons are found immobile at low temperature [23]. At elevated temperatures muon diffusion starts and becomes confined to a limited number of vacant sites within the crystal structure. However, above $\approx 250 \mathrm{~K}$ muon diffuses with an activation energy $\approx 140 \mathrm{meV}$. 
In the present model, muon is assumed to tunnel with an activation energy $140 \mathrm{meV}$. For barrier width $b=2.0 \AA$, the barrier transparency coefficient $\beta$ becomes $1.6 \times 10^{-5}$. Then the jump rate for muon diffusion becomes

$$
\frac{1}{\tau}=1.9 \times 10^{5} T^{3 / 2} \mathrm{e}^{-1624 / T} \quad\left[\mathrm{~s}^{-1}\right] .
$$

This formula is a good fit to the expcrimental results over an approximate temperature range $200-300 \mathrm{~K}$.

$$
\text { 3.8. } N i_{0.35} T i_{0.65} H_{1.5}
$$

Muon diffusion in this intermetallic hydride has been studied over the temperature range $20-335 \mathrm{~K}$ [24]. It is found that the muon is immobile up to $150 \mathrm{~K}$ and at elevated temperatures the motion of muon is strongly related to the mobility of hydrogen atoms present in the hydride. In the temperature range 150-220 K the diffusion of muon remains confined to a limited number of vacant sites so that the diffusion is very small. IIowever, over $220-335 \mathrm{~K}$ the muon diffuses in a tunnelling mode with an activation energy $220 \mathrm{meV}$.

In the present model, the muon is assumed to tunnel with an activation energy $220 \mathrm{meV}$. Then, with the barrier width $b=1.0 \AA$, the value of $\beta$ becomes $10^{-3}$ yielding the jump rate formula

$$
\frac{1}{\tau}=5 \times 10^{6} T^{3 / 2} \mathrm{e}^{-2550 / T}\left[\mathrm{~s}^{-1}\right] .
$$

This formula shows a good fit to the experimental results over 200-335 K.

\section{Discussions}

Due to different hydrogen concentration $x$, the non-stoichiometric hydride passes through different phases offering different hindrance to muon motion. In these phases the muon diffusion is governed by different diffusion parameters and starts at different temperatures. In the present model a single activation energy is used for all such phases of the hydride.

The hydrogen concentration dependence of the jump rate is described by the factor $(v-x)$ which corresponds to the number of vacant hydrogen sites in the lattice structure of the non-stoichiometric hydrides. Different values of the pre-exponential factors in the jump rate formula are exhibited through the different values of $x$.

The author is much grateful and indebted to Prof. D.K. Sinha, Calcutta University, and Prof. P. Bandyopadhyay, Indian Statistical Institute, Calcutta, for many valuable and useful discussions and sugestions on the problem.

\section{References}

[1] H. Sarkar, G.C. Mahato, Acta Phys Pol. A 70, 403 (1986).

[2] G.C. Mahato, Acta Phys. Pol. A 72, 789 (1987).

[3] G.C. Mahato, Acta Phys. Pol. A 77, 597 (1990). 
[4] G.C. Mahato, Acta Plıys. Pol. A 78, 535 (1990).

[5] V.G. Nosov, I.V. Yakovlevs, Nucl. Phys. 68, 609 (1965).

[6] A. Schenck, Muon Rolation Spectroscopy, Adam IIilger Ltd., Bristol 1985, p. 251.

[7] S.F.J. Cox, J. Plıys. C., Solid Slate Phys. 20, 63 (1987).

[8] P. Bandyopadhyay, Lelt. Nuovo Cimento Ser. I 3, 437 (1970).

[9] V.G. Grebinnik, I.I. Gurevich, V.A. Zhukov, A.P. Manych, E.A. Meleshko, L.A. Muratova, B.A. Nikolskii, V.I. Selivanov, V.A. Suctin, Sov. Phys. JETP 41, 777 (1975).

[10] D. Ritcher, R. Hempelmann, O. Hartmann, E. Karlsson, L.O. Norlin, S.F.J. Cox, R. Kutner, J. Chem. Phys. 79, 4564 (1983).

[11] M.R. Choudhury, G.A. Styles, E.F.W. Seymour, S.F.J. Cox, C.A. Scott, G.A. Eatons, J. Pliys., Condens. Malier 1, 4659 (1989).

[12] D. Herlach, V. Claus, K. Furderer, J. Major, A. Seeger, L. Schimnele, M. Schmolz, W. Staiger, W. Templ, E. Yagi, Z. Phys. Chem. (N.F.) 164, 1041 (1989).

[13] F.N. Gygax, A. Schenck, S. Barth, L. Schlapbach, Iyperfine Interact. 31, 407 (1986).

[14] P. Birren, F.N. Gygax, H. IItti, E. Lippelt, A. Schenck, L. Schlapbach, Z. Phys. Chem. (N.F.) 164, 1047 (1989).

[15] W.J. Kossler, H.E. Schone, J.R. Kempton, B. Hitti, C.E. Stronach, G.A. Styles, E.F.W. Seymour, J. Less-Common Met. 129, 327 (1987).

[16] T.T. Phıa, B.J. Beadry, D.T. Peterson, D.R. Torgeson, R.G. Barnes, M. Belhoul, G.A. Styles, E.F.W. Seymour, Phys. Rev. B 28, 6227 (1983).

[17] U.S.W. Schlereth, D. Steinbinder, II. Wipf, Z. Phys. Chem. (N.F.) 164, 929 (1989).

[18] R. Hempelmann, Dr. Richter, O. IIartmann, E. Karlsson, R. Wappling, J. Chem. Phys. 90, 1935 (1989).

[19] A. Baudry, P. Boyer, A. Chikdene, S.W. IIarris, S.F.J. Cox, Iyperfine Interact. 64, 657 (1990).

[20] P. Boyer, A. Baudry, in: Muon Studies in Solid State Physics, Ed. S.F.J. Cox, IOP Publishing Ltd., Bristol 1989, p. 65.

[21] A. Chikdene, A. Baudry, P. Boyer, Z. Phys. Chem. (N.F.) 163, 443 (1989).

[22] A. Baudry, P. Boyer, L.P. Ferreira, S.W. IIarris, S. Miraglia, L. Pontonnier, J. Phys., Condens. Matter 4, 5025 (1992).

[23] S.W. Harris, O. Bartmann, R. IIempclmoun, A.J: Macland, D. Ritcher, C.A. Scott, T. Sundquist, E. Wackelgard, R. Wappling, in: Muon Sludies in Solid State Physics, Ed. S.F.J. Cox, IOP Publishing Ltd., Bristol 1989, p. 73.

[24] R.L. Havill, J.M. Titman, N. Cowlam, IIyperfine Interact. 64, 665 (1990); J. Less-Common Met. 172-174, 770 (1991). 